\title{
Influence of Compressive and Rarefactive Ion-Acoustic Shock Waves in a Multi-Component Degenerate Dense Plasma
}

\author{
P. Halder, and A. A. Mamun
}

\begin{abstract}
The properties of ion-acoustic shock waves associated with the nonlinear propagation of compressive and rarefactive electrostatic perturbations in an unmagnetized collisionless degenerate dense plasma have been investigated theoretically. Our considered model contains degenerate electron, positron, and ion fluids, which are valid for both of the non-relativistic and ultra-relativistic limits. positron, and ion flictic limits. The Burgets and by taking the effect of viscous force in the ion fluid into account. The stationary shock wave solution of Burgers equation is obtained, and numerically analyzed in order to identify the basic properties of ion-acoustic shock structures. It has been shown that depending on plasma parametric values, the degenerate plasma under consideration supports compressive or rarefactive shock structures. It has been also found that the effects of degenerate pressures of electrons, ions, and positrons significantly modify the basic features of the shock waves that are found to exist in such a degenerate plasma. The relevance of our results in astrophysical objects like white dwarfs and neutron stars, which are of scientific interest, are briefly discussed.
\end{abstract}

PACS numbers: 52.27.Ny, 52.35.Fp, 52.35.Tc, 52.25.Vy

Keywords: Ion-acoustic waves, Shock waves, Relativistic effect, Degenerate pressure, Compact objects.

\section{INTRODUCTION}

During the last few decades, significant progress has been taken place to study the linear and nonlinear ionacoustic (IA) waves among the researchers. Interesting nonlinear features of these quantities in different plasma environments as well as their aboundant occurrence in nature has grown the researcher's attention over the past few years. One of the important nonlinear features of the kind is the ion-acoustic shock waves (IASWs). The discovery of remarkable shape-preservation feature of these waves during their interactions by Zabusky et.al in 1965 made their first important applications in communication technology Washimi et.al. showed that such waves, in a weakly nonlinear regime, can be mathematically modeled by the well known shock wave equations. The propagation of the ion-acoustic waves are very important from both the academic point of view and from the view of its vital role in understanding the electrostatic disturbances in space and laboratory plasma. The physics of quantum plasmas, rapidly grown beyond conventional plasmas found in space or laboratory for many years [1, 2] This is mainly due to the potential applications of quantum plasmas in different areas of scientific and technological importance [3-6]. It is a common idea that electronpositron plasmas have presumably appeared in the early universe $[7,8]$ and are frequently encountered in active galactic nuclei [9] and in pulsar magnetospheres [10, 11] This electron-positron plasma is usually characterized as a fully ionized gas consisting of electrons and positrons of equal masses. Recently, there has been a great deal of interest in studying linear as well as nonlinear wave motions in such plasmas $[12,13]$. The nonlinear studies have been focused on the nonlinear self-consistent structures
[12-14] such as envelope solitons, vortices, etc. However, most of the astrophysical plasmas usually contains ions, in addition to the electrons and positrons. Clearly, the properties of wave motions in an electron-positron-ion plasma should be different from those in two-component electron-positron plasmas. For example, Rizzato [15] and Berezhiani et al. [16] have investigated envelope solitons of electromagnetic waves in three-component electronpositron-ion plasmas.

The electron-positron plasmas are thought to be generated naturally by pair production in high energy processes in the vicinity of several astrophysical objects as well as produced in laboratory plasmas experiments with a finite life time [17]. Because of the long life time of the positrons, most of the astrophysical [18] and laboratory plasmas [19] become an admixture of electrons, positrons, and ions. It has also been shown that over a wide range of parameters, annihilation of electrons and positrons, which is the analog of recombination in plasma composed of ions and electrons, is relatively unimportant in classical, [20] as well as in dense quantum plasmas [21] to study the collective plasma oscillations. The ultradense degenerate electron positron plasmas with ions are believed to be found in compact astrophysical bodies like neutron stars and the inner layers of white dwarfs [21$24]$ as well as in intense laser-matter interaction experiments $[25,26]$. Therefore, it seems important to study the influence of quantum effects on dense e-p-i plasmas. Several authors have theoretically investigated the collective effects in dense unmagnetized and magnetized ep-i quantum plasmas under the assumption of low-phase velocity (in comparison with electron/positron Fermi velocity) [27-29]. In these studies, the authors have focused on the lower order quantum corrections appearing in the 
well known classical modes.

A dense plasma is usually characterized as cold and degenerate such as that encountered in metals and semiconductors. However, it has been remarked that a hot fusion plasma such as that found in dense steller objects (e.g., white dwarfs) may also be considered as quantum degenerate plsma [1]. In such environments the production of positrons is and a degenerate plasma of electronpositron-ion can be expected. The main objection to the existence of dense electron-positron-ion plasma may be high electron-positron annihilation rate which is naturally expected where the electron and positron density are very high. In a typical white dwarf star the electron density can be as high as $10^{28} \mathrm{~cm}^{-3}$, however, for massive stars [30] such as that for a collapsing white dwarf, this value can even be much higher [24]. The propagation and collision of small-amplitude ion-acoustic waves in ultra relativistic plasma have been already investigated $[31,32]$.

Now-a-days, a number of authors have become interested to study the properties of matter under extreme conditions [33-36]. Recently, a number of theoretical investigations have also been made of the nonlinear propagation of electrostatic waves in degenerate quantum plasma by a number of authors [37-39] etc. However, these investigations are based on the electron equation of state valid for the non-relativistic limit. Some investigations have been made of the nonlinear propagation of electrostatic waves in a degenerate dense plasma based on the degenerate electron equation of state valid for ultrarelativistic limit $[40,41,43]$.We are interested to study the dissipasion relation of the ion-acoustic waves in a degenerate e-p-i plasma system where we added positrons for the rather long lifetime of positrons, most of the astrophysical $[9,11-13,18,24,44,45]$ as we have mentioned in the introductory chapter. The ion-acoustic waves are longitudinal oscillations of the ions (and the electronpositron) a dusty e-p-i plasma. The linear dispersion relation (DR) will give the relation between the wave frequency ' $\omega$ and the wave number ' $\kappa$. The number density of the degenerate electron and positron in a compact object is so high (e. g. in white dwarfs, the degenerate electron number density can be order of $10^{36} \mathrm{~cm}^{-3}$ even more, and order of $10^{36} \mathrm{~cm}^{-3}$ even more in neutron stars) that the non-relativistic limit is not practically applicable for degenerate fermions (electron-positrons). The equation of state for degenerate particles in astrophysical compact objects is the ultra-relativistic limit mathematically explained by $\mathrm{S}$. Chandrasekhar $[24,46]$. It is very important to note that the degenerate pressure depends only on the fermion number density, but not on its temperature.

To the best of our knowledge, no theoretical investigation has been developed to study the extreme condition of matter for both non-relativistic and ultra-relativistic limits in a degenerate plasma system with the nonlinear propagation and formation of the ion-acoustic shock waves. Therefore, in our present investigation, we consider a degenerate dense plasma system in absence of the magnetic field containing non-relativistic degenerate cold ion fluid, both non-relativistic and ultra-relativistic degenerate electrons and positrons where the ion is the heavier element among all other elements. To study the basic features of the ion-acoustic nonlinear structures of shock wave in such unmagnetized three component degenerate dense plasma, we have studied the Burgers equation and the numerical solution of Burgers equation. The model is relevant to compact interstellar objects (e. g., white dwarf, neutron star, etc.).

\section{GOVERNING EQUATIONS}

We consider an unmagnetized collisionless three component degenerate dense plasma system consisting of non-relativistic degenerate cold degenerate ion fluid and both non-relativistic and ultra-relativistic degenerate electrons and positrons fluids. We assume that the ion is the heavier element among all other considering elements. The dynamics of the one dimensional ion-acoustic waves in such a three component degenerate dense plasma system is governed by

$$
\begin{aligned}
& \frac{\partial n_{s}}{\partial t}+\frac{\partial}{\partial x}\left(n_{s} u_{s}\right)=0, \\
& \frac{\partial u_{i}}{\partial t}+u_{i} \frac{\partial u_{i}}{\partial x}+\frac{\partial \phi}{\partial x}+\frac{K_{1}}{n_{i}} \frac{\partial n_{i}^{\alpha}}{\partial x}-\eta \frac{\partial^{2} u_{i}}{\partial x^{2}}=0, \\
& n_{e} \frac{\partial \phi}{\partial x}-K_{2} \frac{\partial n_{e}^{\gamma}}{\partial x}=0, \\
& n_{p} \frac{\partial \phi}{\partial x}-K_{2} \frac{\partial n_{p}^{\gamma}}{\partial x}=0, \\
& \frac{\partial^{2} \phi}{\partial x^{2}}=n_{e} \alpha_{e}-n_{i}-\alpha_{p} n_{p},
\end{aligned}
$$

where $n_{s}$ is the plasma number density of the species $s$ ( $\mathrm{s}=\mathrm{e}$ for electron, $\mathrm{i}$ for ion, and $\mathrm{p}$ for positron) normalized by its equilibrium value $n_{s o}\left(n_{e 0}\right), u_{s}$ is the plasma species fluid speed normalized by $C_{i m}=\left(m_{e} c^{2} / m_{i}\right)^{1 / 2}$ with $m_{e}\left(m_{i}\right)$ being the electron (ion) rest mass mass and $c$ being the speed of light in vacuum, $\phi$ is the electrostatic wave potential normalized by $m_{e} c^{2} / e$ with $e$ being the magnitude of the charge of an electron, the time variable $(t)$ is normalized by $\omega_{p i}=\left(4 \pi n_{0} e^{2} / m_{i}\right)^{1 / 2}$, and the space variable $(x)$ is normalized by $\lambda_{m}=$ $\left(m_{e} c^{2} / 4 \pi n_{0} e^{2}\right)^{1 / 2}$. The coefficient of viscosity $\eta$ is a normalized quantity given by $\omega_{i} \lambda_{m i}^{2} m_{s} n_{s 0}$, and $\alpha_{e}$ is the ratio of the number density of electron and ion $\left(n_{e} / n_{i}\right)$ and $\alpha_{p}$ is the ratio of the number density of positron and ion $\left(n_{p} / n_{i}\right)$. The constants $K_{1}=n_{0}^{\alpha-1} K_{i} / m_{i}{ }^{2} C_{i}{ }^{2}$ and $K_{2}=n_{0}^{\gamma-1} K_{e} / m_{i} C_{i}{ }^{2}=n_{0}^{\gamma-1} K_{p} / m_{i} C_{i}{ }^{2}$. The equations of state used here for the degenerate pressures of electrons, ions and positrons are given by

$$
P_{i}=K_{i} n_{i}^{\alpha},
$$

where

$$
\alpha=\frac{5}{3} ; \quad K_{i}=\frac{3}{5}\left(\frac{\pi}{3}\right)^{\frac{1}{3}} \frac{\pi \hbar^{2}}{m} \simeq \frac{3}{5} \Lambda_{c} \hbar c,
$$


for the non-relativistic limit (where $\Lambda_{c}=\pi \hbar / m c=1.2 \times$ $10^{-10} \mathrm{~cm}$, and $\hbar$ is the Planck constant divided by $2 \pi$ ). While for the electron fluid,

$$
P_{e}=K_{e} n_{e}^{\gamma}
$$

and while for the positron fluid

$$
P_{p}=K_{p} n_{p}^{\gamma}
$$

where for non-relativistic limit $[33-35,40,43]$

$$
\gamma=\alpha ; K_{e}=K_{p}=K_{i}
$$

and for the ultra-relativistic limit [33-35, 40, 43$]$

$$
\gamma=\frac{4}{3} ; \quad K_{e}=K_{p}=\frac{3}{4}\left(\frac{\pi^{2}}{9}\right)^{\frac{1}{3}} \hbar c \simeq \frac{3}{4} \hbar c,
$$

\section{DERIVATION OF BURGERS EQUATION}

Now we derive a dynamical equation for the nonlinear propagation of the ion-acoustic shock waves by using Eqs. $(1-5)$. To do so, we employ a reductive perturbation technique to examine electrostatic perturbations propagating in the relativistic degenerate dense dusty plasma due to the effect of dissipation, we first introduce the stretched coordinates [47]

$$
\begin{aligned}
\zeta & =\epsilon\left(x-V_{p} t\right), \\
\tau & =\epsilon^{2} t,
\end{aligned}
$$

where $V_{p}$ is the wave phase speed $(\omega / k$ with $\omega$ being angular frequency and $k$ being the wave number of the perturbation mode), and $\epsilon$ is a smallness parameter measuring the weakness of the dispersion $(0<\epsilon<1)$. We then expand $n_{i}, n_{e}, u_{i}$, and $\phi$, in power series of $\epsilon$ :

$$
\begin{aligned}
& n_{i}=1+\epsilon n_{i}^{(1)}+\epsilon^{2} n_{i}^{(2)}+\cdots, \\
& n_{e}=1+\epsilon n_{e}^{(1)}+\epsilon^{2} n_{e}^{(2)}+\cdots, \\
& n_{p}=1+\epsilon n_{p}^{(1)}+\epsilon^{2} n_{p}^{(2)}+\cdots, \\
& u_{i}=\epsilon u_{i}^{(1)}+\epsilon^{2} u_{i}^{(2)}+\cdots, \\
& \phi=\epsilon \phi^{(1)}+\epsilon^{2} \phi^{(2)}+\cdots,
\end{aligned}
$$

and develop equations in various powers of $\epsilon$. To the lowest order in $\epsilon$, using equations (12)-(18) into equations (1) - (5) we get as, $u_{i}^{(1)}=V_{p} \phi^{(1)} /\left(V_{p}^{2}-K_{1}^{\prime}\right)$, $n_{i}^{(1)}=\phi^{(1)} /\left(V_{p}^{2}-K_{1}^{\prime}\right), n_{e}^{(1)}=n_{p}^{(1)}=\phi^{(1)} / K_{2}^{\prime}$, and $\left.V_{p}=\sqrt{(} \frac{K_{2}^{\prime}}{\alpha_{e}-\alpha_{p}}+K_{1}^{\prime}\right)$, where $K_{1}=n_{0}^{\alpha-1} K_{i} / m_{i}{ }^{2} C_{i}{ }^{2}$ and $K_{2}=n_{0}^{\gamma-1} K_{e} / m_{i} C_{i}{ }^{2}=n_{0}^{\gamma-1} K_{p} / m_{i} C_{i}{ }^{2}$. The relation $\left.V_{p}=\sqrt{(} \frac{K_{2}^{\prime}}{\alpha_{e}-\alpha_{p}}+K_{1}^{\prime}\right)$ represents the dispersion relation for the ion-acoustic type electrostatic waves in the degenerate plasma under consideration.

We are interested in studying the nonlinear propagation of these dissipative ion-acoustic type electrostatic waves in a three components degenerate plasma. To the next higher order in $\epsilon$, we obtain a set of equations

$$
\begin{aligned}
& \frac{\partial n_{s}^{(1)}}{\partial \tau}-V_{p} \frac{\partial n_{s}^{(2)}}{\partial \zeta}-\frac{\partial}{\partial \zeta}\left[u_{s}^{(2)}+n_{s}^{(1)} u_{s}^{(1)}\right]=0 \\
& \frac{\partial u_{i}^{(1)}}{\partial \tau}-V_{p} \frac{\partial u_{i}^{(2)}}{\partial \zeta}+u_{i}^{(1)} \frac{\partial u_{i}^{(1)}}{\partial \zeta}+\frac{\partial \phi^{(2)}}{\partial \zeta}-\eta \frac{\partial^{2}}{\partial \zeta^{2}} u_{p}^{(1)} \\
& +K_{1}^{\prime} \frac{\partial}{\partial \zeta}\left[n_{i}^{(2)}+\frac{(\alpha-2)}{2}\left(n_{i}^{(1)}\right)^{2}\right]=0 \\
& \frac{\partial \phi^{(2)}}{\partial \zeta}-K_{2}^{\prime} \frac{\partial}{\partial \zeta}\left[n_{e}^{(2)}+\frac{(\gamma-2)}{2}\left(n_{e}^{(1)}\right)^{2}\right]=0 \\
& \frac{\partial \phi^{(2)}}{\partial \zeta}-K_{2}^{\prime} \frac{\partial}{\partial \zeta}\left[n_{p}^{(2)}+\frac{(\gamma-2)}{2}\left(n_{p}^{(1)}\right)^{2}\right]=0 \\
& 0=\alpha_{e} n_{e}^{(2)}-n_{i}^{(2)}-\alpha_{p} n_{p}^{(2)}
\end{aligned}
$$

Now, combining (19-23) we deduce a Burgers equation

$$
\frac{\partial \phi^{(1)}}{\partial \tau}+A \phi^{(1)} \frac{\partial \phi^{(1)}}{\partial \zeta}=C \frac{\partial^{2} \phi^{(1)}}{\partial \zeta^{2}},
$$

where the value of $A$ and $C$ are given by

$$
\begin{aligned}
& A=\frac{\left(V_{p}^{2}-K_{1}^{\prime}\right)^{2}}{2 V_{p}}\left[\frac{3 V_{p}^{2}+K_{1}^{\prime}(\alpha-2)}{\left(V_{p}^{2}-K_{1}^{\prime}\right)^{3}}\right. \\
& \left.+\frac{(\gamma-2)\left(\alpha_{e}-\alpha_{p}\right) \beta^{2}}{{K_{2}^{\prime}}^{2}}\right], \\
& C=\frac{\eta}{2} .
\end{aligned}
$$

The shock wave solution of (24) is

$$
\phi^{(1)}=\phi_{m}\left[1-\tanh \left(\frac{\xi}{\delta}\right)\right],
$$

where the special stretched coordinates, $\xi=\zeta-u_{0} \tau$, the amplitude, $\phi_{m}=u_{0} / A$, the width, $\delta=2 C / u_{0}, u_{0}$ is the wave speed and the parameter $\eta$ was chosen from standard value [48] for the system under consideration.

\section{NUMERICAL ANALYSIS}

We have numerically solved the Burgers equation(24), and have studied the effects of $u_{0}, \eta, \alpha_{e}$, and $\alpha_{p}$ with $\xi$ on ion-acoustic nonlinear structures of shock waves in both non-relativistic and ultra-relativistic degenerate electrons and positrons where ions always being non-relativistic degenerate. It is obvious from figures 1 - 16 that the degenerate plasma system under consideration supports compressive and reductive ion-acoustic shock waves which are associated with both positive and negative potential and the amplitude of these nonlinear structures of shock waves depend on the Chandrasekhar limits [33], i.e. nonrelativistic and ultra-relativistic limits.

In figures 1 - 8 we have observed the effect of $u_{0}, \eta$ $\alpha_{e}$, and $\alpha_{p}$ on the potential structure with the variation 


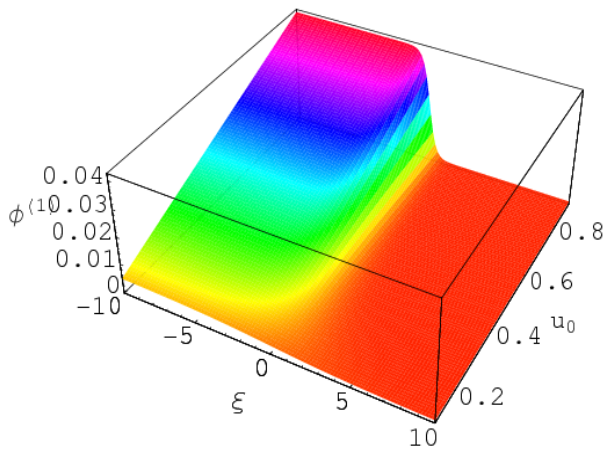

FIG. 1: The effect of $u_{0}$ on shock wave for positive potential when e-i-p being non-relativistic degenerate.

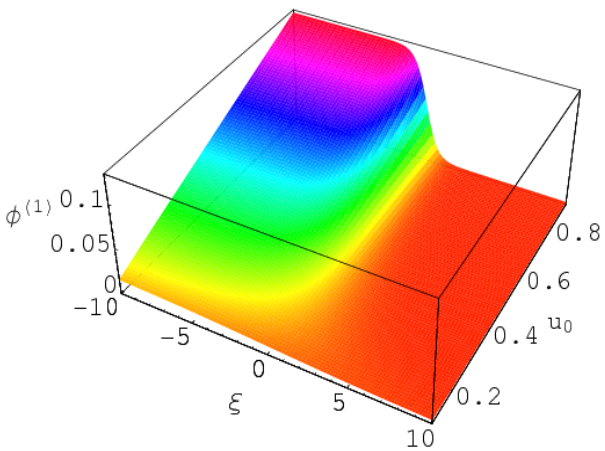

FIG. 2: The effect of $u_{0}$ on shock wave for positive potential when e-p being ultra-relativistic and i being non-relativistic degenerate.

of $\xi$ in case of both non-relativistic and ultra-relativistic limits. It has been observed when the values of $\alpha_{e}$ is greater than $0.62\left(\alpha_{e}>0.62\right)$ then we observe the formation of positive potential for the nonlinear structures of shock wave in our considered degenerate plasma system. Again we consider the values of $\alpha_{e}$ is less than or equal to $0.62\left(\alpha_{e} \leq 0.62\right)$ then we observe the negative potential for the nonlinear structures as shown in figures from 7 to 16. It should be noted here that in both cases we keep all the parameters same so that it could be easy to analysis the effects of every parameter.

To observe the nature of the positive potential in our considered degenerate plasma system, we have studied the effect of the increasing value of $u_{0}, \eta, \alpha_{e}$, and $\alpha_{p}$ on the potential, $\phi^{(1)}$ with the variation of $\xi$ in both limits from figures 1 to 8 . The interesting point is that with

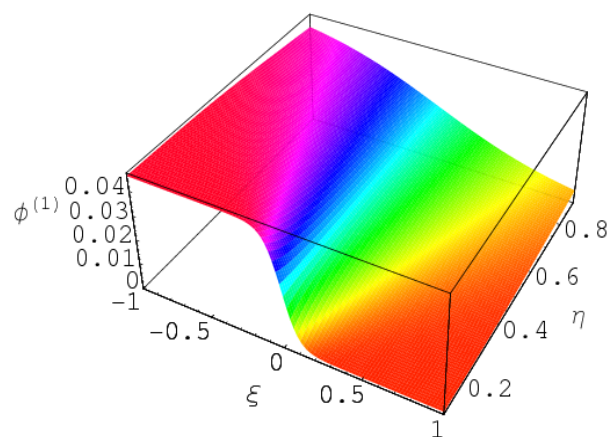

FIG. 3: The effect of $\eta$ on shock wave for positive potential when e-i-p being non-relativistic degenerate.

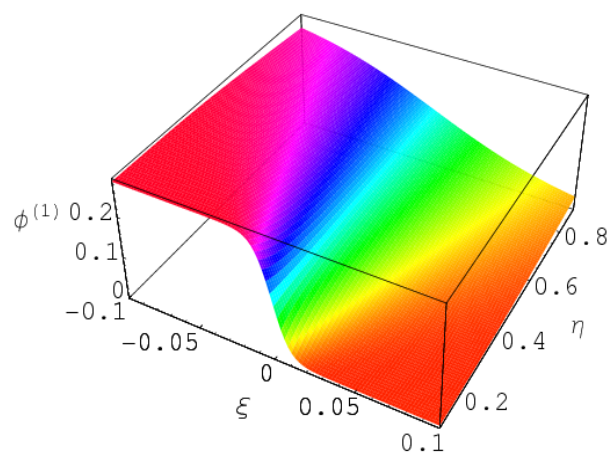

FIG. 4: The effect of $\eta$ on shock wave for positive potential when e-p being ultra-relativistic and i being non-relativistic degenerate.

the increasing values of $u_{0}, \alpha_{p}$, and $\alpha_{e}$, the values of potential increases smoothly, but the increasing values of $\eta$ the potential decreases smoothly. It should be noted that the formation of the compressive ion-acoustic shock waves depends only on the values of the ratio of the electron number density and the ion number density $\left(\alpha_{e}\right)$ and does not depend on the values of $u_{0}$ and $\alpha_{p}$ (the ratio of the electron number density and the positron number density). And the figures $1-8$ also show us that the potential for electron-positron (e-p) being ultrarelativistic and ion (i) being non-relativistic degenerate is always greater than for electron-ion-positron (e-i-p) being non-relativistic degenerate.

From the study of the negative potential in our three components degenerate plasma system, we have observed that with the increasing value of $u_{0}$ and $\eta$ the potential 


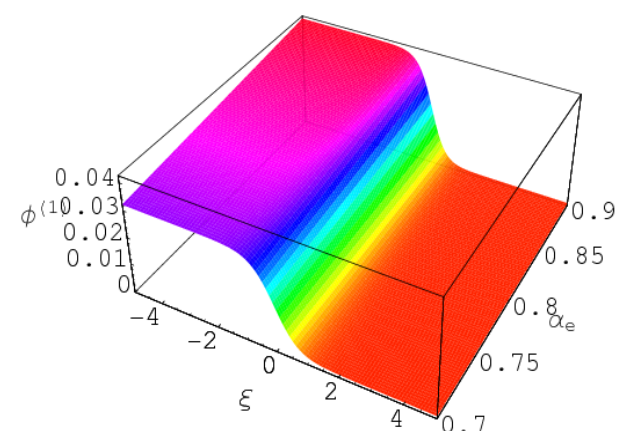

FIG. 5: The effect of $\alpha_{e}$ on shock wave for positive potential when $\mathrm{e}-\mathrm{i}-\mathrm{p}$ being non-relativistic degenerate.

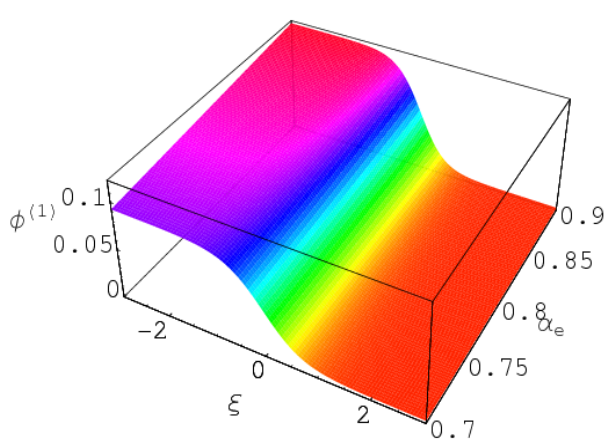

FIG. 6: The effect of $\alpha_{e}$ on shock wave for positive potential when e-p being ultra-relativistic and i being non-relativistic degenerate.

also increases. But it has been also noted that with the increasing values of $\alpha_{e}$ and $\alpha_{p}$, the potential, $\left(\phi^{(1)}\right)$ also decreases very smoothly in both limits from figures 7 to 16. It should be noted here that in this case, the values of $\alpha_{e}$ is always less than or equal to $0.62\left(\alpha_{e} \leq 0.62\right)$. It needs to be pointed here that the formation of the reductive ion-acoustic shock waves (shown in figures 7 to 16) depends only on the values of the ratio of the electron number density and the ion number density $\left(\alpha_{e}\right)$ and does not depend on the values of $u_{0}$ and $\alpha_{p}$ (the ratio of the electron number density and the positron number density). From the analysis of the negative potential structures for $\alpha_{e} \leq 0.62$ (shown in figures 7 16) it has been again pointed out that the potential for electron-positron (e-p) being ultra-relativistic and ion (i) being non-relativistic degenerate is always greater than

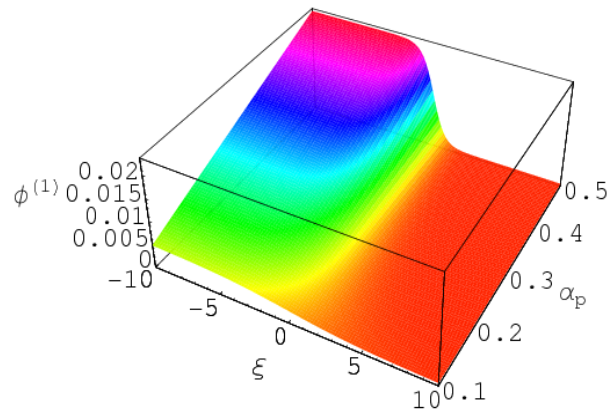

FIG. 7: The effect of $\alpha_{p}$ on shock wave for positive potential when e-i-p being non-relativistic degenerate.

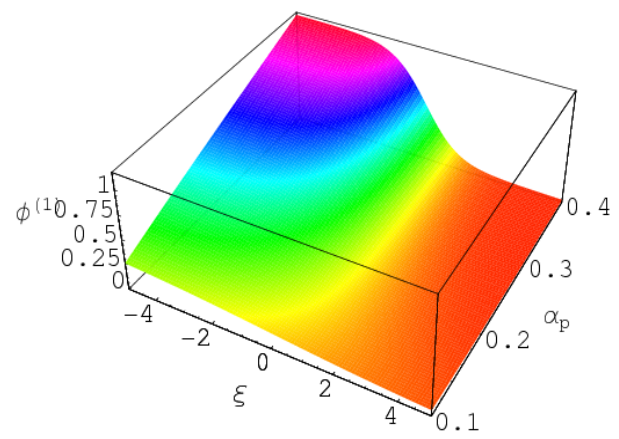

FIG. 8: The effect of $\alpha_{p}$ on shock wave for positive potential when e-p being ultra-relativistic and i being non-relativistic degenerate.

for electron-ion-positron (e-i-p) being non-relativistic degenerate.

It is to be noted here that we have taken all the parameters in normalized form, so all the ranges of parameters are taken arbitrarily.

\section{DISCUSSION}

We have considered an unmagnetized degenerate dense plasma containing non-relativistic degenerate cold ions fluid and both non-relativistic and ultra-relativistic degenerate electrons and positrons fluid, and have examined the basic features of the electrostatic nonlinear structures that are found to exist in such degenerate 


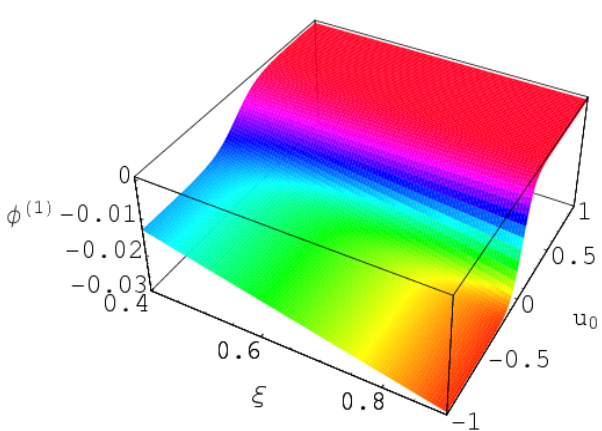

FIG. 9: The effect of $u_{0}$ on shock wave for negative potential when $\mathrm{e}-\mathrm{i}-\mathrm{p}$ being non-relativistic degenerate.

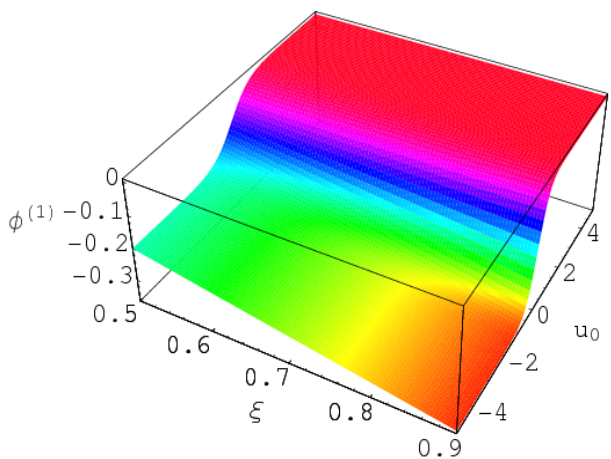

FIG. 10: The effect of $u_{0}$ on shock wave for negative potential when e-p being ultra-relativistic and i being non-relativistic degenerate.

dense plasma. In our present investigation all the degenerate constitutes of the considered dense unmagnetized plasma system follow relativistic limits of pressure. The nonlinear IA shock waves and its propagation have been described thoroughly with Burgers equation (24) and it's solution (27). The effects of different plasma parameters on the nonlinear propagation of IA shocks waves have been graphically shown (figures 1 - 12).

The profiles of shock wave, caused by the balance between nonlinearity and dissipation, are depicted in figures 1-12. And the potential of the ion-acoustic shock waves profiles for non-relativistic degenerate ions fluid and ultra-relativistic degenerate electrons and positron fluid is different from that when all the particles follow the same limit. From the mathematical calculation and the numerical solution of Burgers equation we have

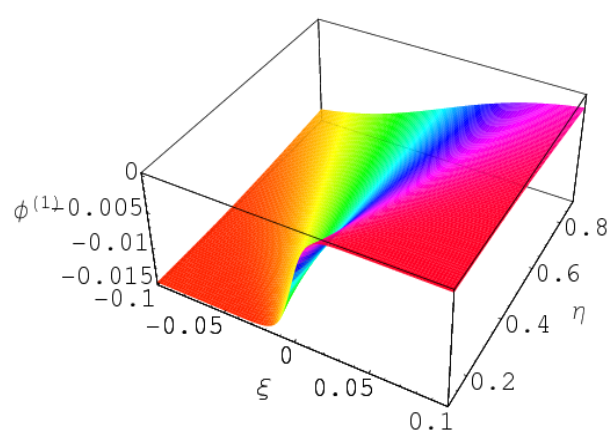

FIG. 11: The effect of $\eta$ on shock wave for negative potential when $\mathrm{e}-\mathrm{i}-\mathrm{p}$ being non-relativistic degenerate.

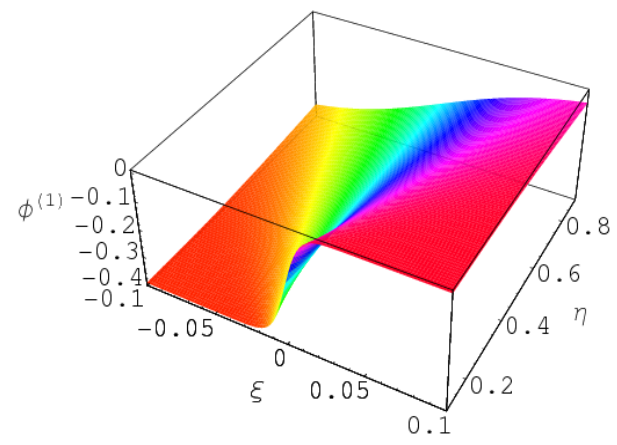

FIG. 12: The effect of $\eta$ on shock wave for negative potential when e-p being ultra-relativistic and i being non-relativistic degenerate.

found that for a certain value of $\alpha_{p}$ we get both positive and negative potential when all other parameters are kept same, i.e. when the values of $\alpha_{p}$ is always greater than $0.62\left(\alpha_{p}>0.62\right)$ then we obtain positive potential and when the values of $\alpha_{p}$ is less than or equal to 0.62 $\left(\alpha_{p} \leq 0.62\right)$ then we obtain negative potential. It has made the great interest in the study of the ion-acoustic nonlinear structures of shock waves in an unmagnetized degenerate dense plasma system to analysis the existence conditions for the positive and negative potential where the elements, electrons, ions, and positrons are always being degenerate.

Our present investigation is different from the related investigations $[31,40,41,43,49]$ in the way that we have considered the pressure of all the constituent particles (electrons and ions), as the whole system is degen- 


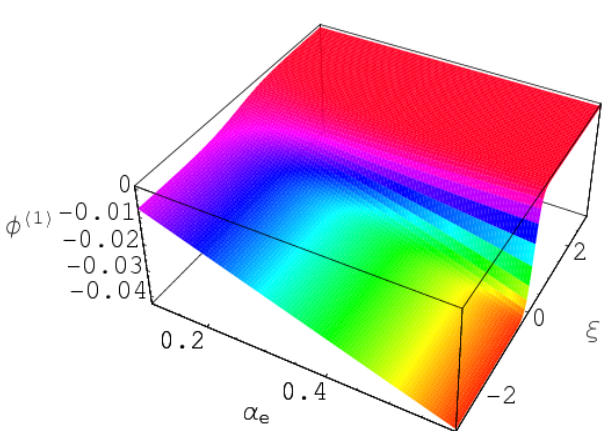

FIG. 13: The effect of $\alpha_{e}$ on shock wave for negative potential when e-i-p being non-relativistic degenerate.

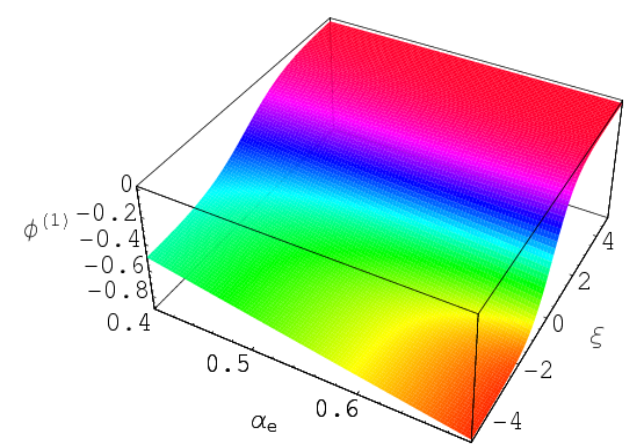

FIG. 14: The effect of $\alpha_{e}$ on shock wave for negative potential when e-p being ultra-relativistic and i being non-relativistic degenerate.

erate and all the particles should follow the equation of state $(6)-(11)$ whatever the limit is (non relativistic or ultra-relativistic). This is obvious that the shock profiles obtained from our present investigation are also quite different from the previous investigation $[31,40,41,43,50]$ in the sense that we have had electrostatic shock profiles while the others have got to support electrostatic solitary profiles $[40,41,43]$ and double layers $[40,41]$. The degenerate dense plasma is found to support shock structures whose basic features dependent on the plasma number density. From this point of view our present investigation is more acceptable and the system constituents have made the validity of our investigations unique

We note that in our numerical analysis we have used a wide range of the degenerate plasma parameters [31$33,40,43$, which are relevant for many cosmic environ-

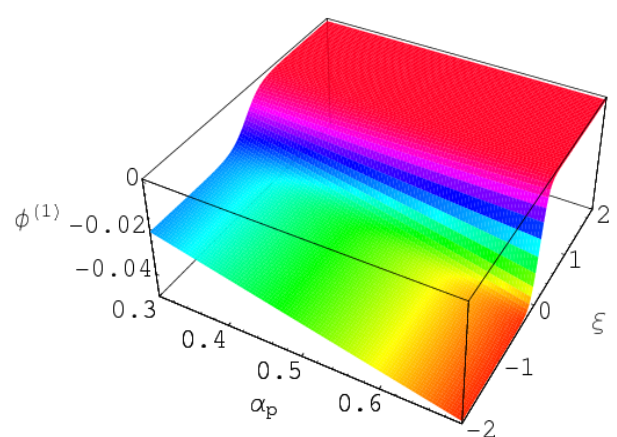

FIG. 15: The effect of $\alpha_{p}$ on shock wave for negative potential when e-i-p being non-relativistic degenerate.

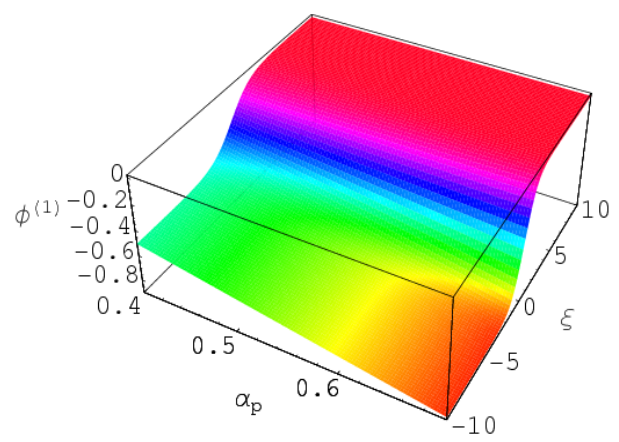

FIG. 16: The effect of $\alpha_{p}$ on shock wave for negative potential when e-p being ultra-relativistic and i being non-relativistic degenerate.

ments and compact astrophysical objects. The results of the present investigation is, therefore, expected to be useful in understanding the dispersion properties of the electrostatic shock waves in such cosmic environments $[9,11,18]$, compact astrophysical objects $[12,13,24]$ and interstellar compact objects $[44,45]$. The electrostatic waves in an ultra-relativistic and non-relativistic degenerate dense plasma, which is relevant to interstellar compact objects like white dwarfs, have been investigated. The results, which have been found from this investigation, represent ion acoustic-type of electrostatic waves in which the restoring force comes from the electron-ion degenerate pressure and inertia is provided by the ion mass density.

It can be expected that the basic features and the underlying physics of the IA shock waves with the exis- 
tence conditions for positive and negative potential, that have been presented in our present work should be verified by further laboratory experiments. It may, therefore, be proposed to perform a laboratory experiment which will be able to identify the special new features of the IA shock waves in a three components degenerate dense plasma system that have been predicted in this investigation. It may also be added here that our investigation is valid for small amplitude IA shock waves and for unmagnetized and uniform degenerate dense plasma system. These results may be useful to explain some aspects of shock waves in dense e-p-i degenerate plasmas. However, arbitrary amplitude IA shock waves in uniform/nonuniform three component degenerate plasma with or without the effects of dust and external magnetic field are also problems of recent interest for many space and laboratory dusty plasma situations, but beyond the scope of our present investigation. Although such plasmas cannot be produced in a laboratory, yet they are gaining considerable attention of the researchers working on dense astrophysical plasmas and numerical simulations. We hope that our present investigation will be helpful for understanding the basic features of the localized electrostatic disturbances in compact astrophysical objects (e.g. white dwarfs, neutron stars, black hole, etc.).

\section{ACKNOWLEDGMENTS}

P.Halder is profoundly grateful to the Ministry of Science and technology (Bangladesh) for awarding the National Science and Technology (NST) fellowship.

\section{REFERENCES}

[1] G. Manfredi, Fields Inst. Commun. 46, 263 (2005).

[2] M. Bonitz et al., Introduction to Quantum Plasmas, in: Introduction to Complex Plasmas (Springer, Berlin, 2010).

[3] A. Markowich et al., Semiconductor Equations (Springer, Vienna, 1990).

[4] G. Agrawal, Nonlinear Fiber Optics (Academic, San Diego, 1995)

[5] W. Li et al., Phys. Rev. Lett. 94, 173001 (2005).

[6] D. Kremp et al., Phys. Rev. E 60, 4725 (1999).

[7] W. Misner et al., Gravitation, (Freeman, San Francisco, 1973), p. 763 .

[8] M. J. Rees, The Very Early Universe, Cambridge University Press, Cambridge, 1983).

[9] H. R. Miller and P. J. Witta, Active Galactic Nuclei, (Springer-Verlag, Berlin, 1987), p. 202.

[10] P. Goldreich and W. H. Julian, Astrophys. J. 157, 869 (1969).

[11] F. C. Michel, Rev. Mod. Phys. 54, 1 (1982).

[12] K. Shukla et al., Phys. Rep. 135, 1 (1986).

[13] M. Y. Yu et al., Astrophys. J. 309, L63 (1986).

[14] F. C. Michel, Theory of Neutron Star Magnetospheres(Chicago University Press, Chicago, 1991)

[15] F. B. Rizzato, Plasma Phys. 40, 289 (1988).

[16] V. I. Berezhiani et al., Phys. Rev. E 50, 448 (1994).

[17] M.K. Mishra et al., Phys. Rev. E 76, 036401 (2007).

[18] E. Tandberg-Hansen and A. G. Emslie, The Physics of Solar Flares (Cambridge University Press, Cambridge, 1988), p. 124 .

[19] R. G. Greaves and C. M. Surko, Phys. Rev. E 75, 3846 (1995).

[20] C. M. Surko and T. J. Murphy, Phys. Fluids B 2, 1372 (1990).

[21] S. Ali et al., Phys. Plasmas 14, 082307 (2007).

[22] D. Lai, Rev. Mod. Phys. 73, 629 (2001).

[23] A.K. Harding and D. Lai, Rep. Prog. Phys. 69, 2631 (2006).

[24] S. L. Shapiro and S. A. Teukolsky, Black holes, White dwarfs and neutron Stars: The Physics of Compact objects (John Wiley and Sons, New York, 1983).

[25] V.I. Berezhiani et al., Phys. Rev. A 46, 6608 (1992)

[26] I.G. Lebo and V.F. Tishkin, Hydrodynamic Instabilities in ICF Problems (Fizmatlit, Moscow, 2006).

[27] A. Mushtaq and S. A. Khan, Phys. Plasmas 14, 052307 (2007).

[28] S. A. Khan and W. Masood, Phys. Plasmas 15, 062301 (2007).

[29] H. Ren et al., J. Phys. A: Math. Theor. 41, 11501 (2007).

[30] S. Chandrasekhar, Science 226, 4676 (1984).

[31] M. Akbari-Moghanjoughi, Phys. Plasma 17, 082315 (2010).

[32] M. Akbari-Moghanjoughi, Phys. Plasma 17, 072306 (2010).

[33] S. Chandrasekhar, Mon. Not. R. Astron. Soc. 170, 405 (1935).

[34] S. Chandrasekhar, Phil. Mag. 11, 592 (1931).

[35] S. Chandrasekhar, Astrophys. J. 74, 81 (1931).

[36] S. Chandrasekhar, The Observatory 57 (1934).

[37] F. Hass, Phys. Plasmas 13, 042309 (2007).

[38] A. Misra and S. Samanta, Phys. Plasmas 15, 123307 (2008).

[39] A. P. Misra et al. Phys. Plasmas 17, 032307 (2010).

[40] A. A. Mamun and P. K. Shukla, Phys. Plasmas 17, 104504 (2010).

[41] N. Roy et al. Phys. Plasmas 19, 033705 (2012).

[42] P. Halder et al. International Journal Research Develop. Organisation 4, 3855-0154 (2015).

[43] A. A. Mamun and P. K. Shukla, Phys. Lett. A 324, 4238 (2010).

[44] L. O. Silva et al., Phys. Rev. Lett. 83, 2703 (1999).

[45] J. Hoyos et al., Astron. Astrophys. 287, 789 (2008).

[46] D. Koester and G. Chanmugam, Rep. Prog. Phys. 53 (1990).

[47] S. Maxon and J. Viecelli, Phys. Rev. Lett. 32, 4 (1974).

[48] A. Gavrikov et al. 32nd EPS Conference on Plasma Physics Tarragona, Russia, 29C,O-2.011 (2005).

[49] S. A. Khan et al., Commun. Theor. Phys. 55, 151 (2011).

[50] M. Lontano et al., Phys. Plasmas 9, 2562 (2002). 